\title{
FREE DISCUSSION ON PROBLEMS OF THE MAGELLANIC CLOUDS
}

\section{Composition and Evolutionary History of the Magellanic Clouds}

Arp: In the colour-magnitude diagram of NGC 330 it may well be that the four stars which Feast has indicated are composite or for some other reason need to be moved bluer in the diagram. The general aspect of the diagram remains very much the same, however, and the problems of interpreting the diagram also remain very much the same. Despite the difficulty of photometry in very tight clusters, however, clusters such as NGC 330 and NGC 458 were deliberately chosen by me because of the field star problem which has been rather loosely alluded to throughout the last few days. In these tight clusters the most important point, that almost all the stars in the various parts of the diagram are cluster members, is most rigorously filled.

In all the publications of these diagrams, moreover, adjacent Small Magellanic Cloud fields were also analysed and confirmed that there were negligible contributions of non-cluster stars. An additional point which does not seem to have been fully appreciated is that these field regions were very small and close to the central regions of the SMC-where the SMC members to a large extent overwhelmed the galactic foreground stars. The individual cluster diagrams were superposed and form a diagram very similar to the SMC field population, indicating again that for these published results the field stars were not a serious problem and that the composition of this region of the SMC could be well understood in terms of the stars of different ages as represented in its clusters.

The second point deals with the fact that I purposely steered away from the large, loose clusters because it was not clear to me that stars in different regions of these clusters were all formed at the same instant. In fact I think we can well understand the results of Herstmonceux and Hodge, Westerlund, and Bok on the large open clusters. Suppose the stars in these clusters were $10^{7}$ to $10^{6}$ years old. We could see a concentration of blue stars in the unevolved main sequence, and several magnitudes brighter just a few evolved giants scattered about-as above. In a tight cluster if the stars are all the same age, say $5 \times 10^{6}$ years-then one gets a well-delineated evolutionary track such as in NGC 330 and NGC 458 where the wellpopulated blue main sequence is as bright as or brighter than the evolved giants.

The question which seems critical to me is why there is such a discrete period of star formation in the tight clusters. Or, put another way, can we locate in the loose clusters where star formation is going on presently and a longer time ago and discover what is different physically about these regions?

Feast: In connection with Arp's remarks on NGC 330 I should like to point out again that there is now no good reason (as discussed in my paper on NGC 330) for assuming that this young blue globular cluster has a metal-poor composition. If there is a small metal deficiency (as Aller and Faulkner's work on nebulae might suggest) one can only say that at the present moment neither the spectroscopy nor the photometry has detected it. 
de Vaucouleurs: In our discussions of ages of clusters, of associations, and of various types of stars we should always keep in mind the very considerable differences in structure, colour, and composition of different sections of the Clouds. For the Large Cloud in particular the youngest supergiant stars are concentrated in the inner spiral arms or arcs which are rich in hydrogen. In the faint outer loop in which Dr. Westerlund has now discovered concentrations of carbon stars and clusters, there is little or no neutral hydrogen, few or no young stars should be seen in this region, which in fact is not resolved until the 15th magnitude. The corresponding region in the Small Cloud is the faint south-preceding wing.

Gascoigne: I would like to quote the colour-magnitude arrays for NGC 1810 (colour-magnitude array published by Sir Richard Woolley) and NGC 2164 (unpublished data); neither of these seems to fall into Arp's scheme.

Woolley: Like at least one speaker yesterday I am a little worried about Professor Bok's interpretation of NGC 1929 as an expanding association. There is a large mass of hydrogen to be seen in one place. If it has always been together, and has always been able to manufacture stars, then certainly some old stars should be seen embedded in it unless they were formed with the velocity of escape from the hydrogen. I am not at all clear why the stars should be formed with the velocity of escape unless the hydrogen itself gave an escape velocity to these stars; and if the internal motions of the hydrogen exceed the velocity of escape, what keeps the hydrogen together? Magnetic forces? If so, can these be looked for perhaps by looking for polarization?

Is it necessary to suppose that the hydrogen has always been "together" and if so is it necessary to suppose that the hydrogen has always been equally capable of forming stars? Is there not reason to suppose that star formation proceeds, at least sometimes, in fits and starts?

We know that on rare occasions the conditions are just right for the formation of a globular cluster. We also know that this is a rare event because most stars are not in fact found in globular clusters but are formed (more or less) free. Is this a case? Will all, or some of it, go into something like NGC 1818?

Without reference books or time to work it out I am not too happy about the application of the virial theorem. The $V^{2}$ given by the theorem is not itself the velocity of escape; to calculate this one must surely have a mass distribution.

Ambartsumian: I should like to stress the importance of the investigations of the type presented here by Professor Bok's paper yesterday for the most fundamental problem standing before us, i.e. for finding an answer to the question if the stars really are being formed from the gaseous masses. Thus I consider it very important to use the possibilities of the investigation of the connections between the young stars and the gaseous clouds.

It is also very essential to investigate in more detail the blue globulars, especially to find more exact information about their connection with diffuse matter.

Bok: A possible further hypothesis-which I did not present-is that the star association NGC 1929 is a star group passing by chance through a large cloud of HIfound by McGee to have a very high mass. This cloud is apparently partially ionized, 
as is shown by Mathewson and by the presence of optical HII regions. Within the star group itself the maximum velocity dispersion could be very much less than $\pm 8 \mathrm{~km} / \mathrm{sec}$ and the group would still be an unstable one. But, whatever the dynamics of the system may be, we cannot really begin the analysis until we have for the field of NGC 1929 stellar radial velocities to $m=13$ and also nebular radial velocities and dispersions very much like those determined by Feast and Courtès for 30 Doradus.

Westerlund: I agree with Arp that in the main body of the Small Magellanic Cloud a colour-magnitude diagram of the type that he has found must be correct for the field stars. However, I do not expect the wing to show many red or yellow Cloud members. NGC 2092 agrees in stellar distribution with NGC 1810. In NGC 2100 the inner parts show brighter blue and red stars than the outer regions.

\section{Period-Luminosity Relationships for Cepheids in the Magellanic Clouds}

de Vaucouleurs: Do students of the period-luminosity relation know of any good reason why the relation has to be a straight line? If there is in fact a slight curvature as both the old Harvard data and the new data suggest, lack of agreement between various determinations of the slope may be due in part to the fact that they refer to different period ranges.

Eggen: Some years ago Kukarkin found two intersecting lines.

Rodgers: The distribution of longer-period cepheids seems to show a displacement from the bar of the SMC. In reply to Ambartsumian, I have found no HII regions near NGC 330 .

Arp: The simplest picture which I can make for the cepheids in the Magellanic Clouds at this time is the following: postulate that the short-period faint cepheids in the NGC 371 field may have slightly more reddening than the long-period cepheids. In that case the slope of the SMC period-luminosity relation could be quite close to the LMC slope. The remaining difference in the 340 points could then be due to the LMC being several tenths of magnitude closer than the SMC.

The apparent moduli of the NGC 371 area cepheids is between $18 \cdot 8$ and 19.0. If the RR Lyrae stars in the Magellanic Clouds have the same internal absorption as in the NGC 371 field (about $A_{B}=0.5 \mathrm{mag}$ ) then the RR Lyraes would appear at $B=19 \cdot 3$ to $19 \cdot 5$, which is in the range observed. If the RR Lyrae stars in the Magellanic Clouds are not reddened, however, and if they are like the RR Lyrae stars in our own Galaxy, then the classical cepheids would need to be almost half a magnitude brighter than in our own Galaxy.

Thackeray: If the SMC is a strongly tilted plane system, then the concentration of the brightest cepheids all down one side of the Cloud will give rise to an error of a few tenths of a magnitude at the bright end of the period-luminosity relation.

Oort: Is there a gap in the distribution of the colours of globular clusters in the Clouds? (By globular clusters I mean all rich clusters in the Clouds.) If there is such a gap, can this be reconciled with the theory that these clusters were formed continually during the entire life of the clouds? 
Tifft: Natural selection has led us to study mostly the extreme blue or extreme red clusters-there may well be many intermediate age clusters we have not yet studied.

Lindblad: If I remember rightly the presence of the blue globular clusters caused some trouble when compared in size and magnitude with the globular clusters in our system. Is there a relation between the colour and the absolute magnitude (luminosity) of the clusters? This would be of interest from the evolutionary point of view.

Gascoigne: We have photometric measurements for about 20 "globular" clusters in the Galaxy, selected on the basis chiefly of apparent magnitude. A colour-magnitude array of these separates them fairly well into two groups, with $B-V$ colours (speaking from memory) from about 0.0 to 0.2 and 0.5 to 0.8 . The scarcity of clusters in the 0.2 to $0.5 \mathrm{~m}$ range appears real.

Arp: In answer to Dr. Lindblad's and Dr. Oort's questions, I would like to point out that as a so-called "blue globular cluster" evolves it gets fainter. In $10^{10}$ years it would get 4 magnitudes fainter. These clusters would become very inconspicuous as they grew fainter. In globular clusters as we ordinarily use the term in our own Galaxy, stars evolve through the $M_{V}=-3$ region, however, and would be easily seen in the Magellanic Clouds. This is the explanation usually given for the dichotomy in distribution between the red and blue clusters in the Magellanic Clouds.

\section{Radio Results for the Small Magellanic Cloud}

Bolton: For the sake of promoting discussion, I would suggest that the two "emission peaks" do not represent separate emitting regions but that they are one broad feature and the dip between them is due to self-absorption by cooler material on the near side of the Cloud. A model of this type would involve velocity dispersion and cloud temperature decreasing from the centre outwards. There is some evidence for such self-absorption in our own Galaxy. Radhakrishnan found one area near IC 443 where strong self-absorption was associated with a minor dust cloud.

Burke: At the Carnegie Institution we surveyed the absorption feature reported by Radhakrishnan, and from our observations we concluded that this feature could equally well be interpreted as a superposition of emission regions, with no absorption. In the case of the SMC, from the picture that we have of two elliptical emission regions, it seems hard to visualize the double peaks as an absorption phenomenon.

Davies: I have found self-absorption in limited areas of our Galaxy which were coincident in position with dense dust clouds. An understanding of the dynamics of the Magellanic Clouds requires a study of the angular distribution of the various velocity components.

Kerr: Self-absorption is certainly a theoretical possibility, but seems an unlikely explanation for such a large-scale phenomenon. There would need to be a widespread region of high-density, low-temperature gas, and the velocity dispersion would need to be very high in the original emitting gas. Also, no analogous region of large-scale self-absorption is known in the Galaxy. 
Oort: If there would be so dense and extensive a mass of very cool hydrogen, one would expect to get also a heavy optical obscuration, of which there is no evidence in the Magellanic Clouds.

Hindman: Bolton's suggestion is attractive to me from the point of view that it would enable us to show that temperature differences exist in the interstellar gas, but the profile shapes, especially where they degenerate to single peaks, would require an increase in velocity dispersion of $2: 1$ with a temperature decrease. Aesthetically, I also find it harder to believe in a very peculiar-shaped low-temperature cloud than in two fairly regular objects.

Burke: There certainly do exist some self-absorption features in the sky, notably the absorption dip near zero velocity observed over a wide range of galactic longitude and latitude. For the benefit of the optical astronomers, the slide shows a hydrogen-line profile taken in this region, showing the very sharp dip that can be interpreted only as absorption by a hydrogen cloud whose temperature is lower than the temperature of the background hydrogen.

Westerhout: The main point in the self-absorption in the centre region is that the dip seen against the bright background in the plane changes into a peak at exactly the same frequency when you go away from the plane and the background disappears. This is clear evidence for a cool foreground cloud. It is exactly the opposite to what Mr. Hindman sees. I see no way to support Mr. Bolton's suggestion at all.

Thackeray: Mr. Hindman's slide the other day showed a rotation curve for the lower sausage-shaped cloud covering the $\mathrm{SMC}$ with a range of about $60 \mathrm{~km} / \mathrm{sec}$. If an interpretation in terms of absorption is possible it would certainly offer a way out from an otherwise puzzling situation. Secondly, is there any possibility of such a large mass of hydrogen in the galactic foreground at low temperature?

Hindman: There is no reason why there should not be such a foreground cloud at low temperature but the coincidence in velocity is highly unlikely.

Feast: Is it not true that the evidence for the separation of the two main $21-\mathrm{cm}$ "objects" depends on assuming that the rotation curve of the flattened component is symmetrical? This may not be so (compare the difficulties about the rotation centre in the LMC). If we adopt the view that the rotation curve can be asymmetrical then it might be possible to explain the SMC as consisting of a flattened rotating disk plus a superimposed halo of HI. Can Mr. Hindman say if such a model could be fitted to the observations?

Hindman: There will always be a velocity difference in the line of sight between the two proposed distributions and this cannot be explained by rotation, but only by expansion or contraction in the line of sight.

Gascoigne: Professor Oort raised the point that there is not much dark matter in the Small Magellanic Cloud; we ought to remember that Wesselink could not see any galaxies near the Cloud, which indicates that there is a lot of dark matter at some place along the line of sight.

Kerr: The argument that the two features are separated in the line of sight does not depend on the velocity difference only, but also on the different distributions of the two over the sky. 
A more detailed comparison between dust (as indicated by Galaxy counts) and neutral hydrogen would be of great interest. One of the HI features seems to be related to the main stellar body; the other entity might contain fewer stars, but more dust.

Mathewson: I would like to remark on the extremely complex structure of the $408 \mathrm{Mc} / \mathrm{s}$ isophotes which are observed in the region of the Small Cloud compared with the Large Cloud region; also the $408 \mathrm{Mc} / \mathrm{s}$ radiation from the vicinity of the SMC has a linearly polarized component-these features observed in the continuum emission may in some way be associated with the region producing the $\mathrm{H}$-line results of Mr. Hindman.

\section{Chemical Composition of the Magellanic Clouds}

Aller: The helium/hydrogen ratio may be established more accurately from gaseous nebulae than from stars. The difficulty comes not from the physics but from the geometry. If the gas has a filamentary structure and stratification also exists one may encounter difficulties. Therefore we should measure the $\mathrm{H} / \mathrm{He}$ ratio at several points in a nebula such as 30 Doradus and in several other nebulae as well. Such a program would require a fair investment of time on a telescope equipped with a nebular spectrograph (not necessarily a 74-inch-a 36 -inch would do). The $\mathbf{O} / \mathrm{H}$ and $\mathrm{Ne} / \mathrm{H}$ ratios are less securely established since $\mathrm{O}$ and $\mathrm{Ne}$ may exist in several ionization stages. The evidence suggests a slightly lower $\mathrm{O} / \mathrm{H}$ and $\mathrm{Ne} / \mathrm{H}$ ratio in the Magellanic Clouds than in our galactic neighbourhood (Orion nebula). Carbon and nitrogen should be investigated. Unfortunately, 30 Doradus and its counterpart, NGC 604 in the Triangulum spiral, have a lower surface brightness than Orion, so we cannot observe the CII $\lambda 4267$ line.

A metal/hydrogen ratio differing from that of the Galaxy by about $30 \%$ would be almost impossible to detect from clusters or from single stars; one cannot determine the temperature of a star accurately enough. Yet changes of this order may influence the evolutionary track of stars and wreak havoc on some of our comparisons. We are a long way from getting stellar compositions from their evolutionary tracks, however.

Eggen: I should like to make two comments here: (1) there is some evidence from stars in our own Galaxy that there is a large spread in the $\mathrm{H} / \mathrm{He}$ ratio in the Galaxy itself, and (2) perhaps one way of obtaining this ratio in the Magellanic Clouds would be from mass determinations by using known eclipsing stars among the brighter Magellanic Cloud stars.

Oort: In connection with the composition of the Magellanic Clouds, I find it very difficult to understand why the abundance of heavier elements in the Clouds is so nearly the same as that in the Galactic System. If one thinks that the heavier elements in our own Galaxy were formed practically completely in the earliest part of its evolution and if one considers that this evolution must clearly have been greatly different from that of the Magellanic Clouds, one wonders how such equality in composition can be understood.

de Vaucouleurs: If one examines large numbers of photographs of spiral galaxies he is soon impressed by the frequency of magellanic-type systems associated 
with large late-type spirals. A striking example is NGC 1232 where the small companion at the edge of the spiral pattern is an almost exact miniature of the Large Magellanic Cloud. Is it possible that such systems are forming out of and escaping from a larger spiral galaxy? In the case of the Magellanic Clouds this hypothesis has been discussed by Mrs. Gaposchkin and Idlis.

Oort: This was probably not the case in our system as the Magellanic system is too far removed from our own Galaxy.

Haro: Blue stars in the galactic halo indicate that this halo extends to distances of the order, at least, of the distance of the Magellanic Clouds.

Tifft: Regarding the galactic halo haze, I would expect it to be something very generally distributed and not connected just with a specific "bridge" between the Galaxy and the Clouds. It may also be noted that in the NGC 121 region where no Cloud blue stars are present for confusion no really blue stars of the type Dr. Haro has mentioned are seen brighter than $V \approx 20$ in a sample of perhaps 1000 stars.

\section{Polarization in the Large Magellanic Cloud}

Visvanathan: The interstellar polarization obtained during the last few months with a single channel photoelectric polarimeter are presented. All the observations were made at the Cassegrain of the 26-inch Mt. Bingar telescope.

In the LMC, of 10 stars observed, one is unpolarized, four are polarized less than $1 \%$, and five have polarization between 1 and $2 \%$. In the Small Cloud, of the eight stars observed, one is unpolarized, and four are polarized less than $1 \%$. All the stars observed are early supergiants selected from the Cloud members list, published by Thackeray, Feast, et al. The integrated radiation observed through a 108-inch aperture in two regions of the Large Cloud shows polarization of 3 and $5 \%$.

In both the Clouds, the position angle of the angle of polarization shows striking parallelism along the bar of the Clouds. In the SMC the position angle varies from 6 to $50^{\circ}$ while in the LMC it varies from 110 to $145^{\circ}$. It is highly significant that such large uniform alignment is found in these irregular galaxies.

The above results should be considered highly preliminary as each star has been observed only once. However, the above observations show the presence of polarization and thereby the presence of magnetic fields in the Clouds.

Recently R. D. Wolstencroft reported 24 and $33 \%$ polarization in the Small and Large Cloud respectively. The above results show the polarization measured with a solid angle of $4^{\circ}$, and as such it represents polarization due both to diffraction scattering and absorption, while our results represent only polarization due to absorption. However, the difference between the Stromlo results and polarization values suggested by Wolstencroft appears to be too great. 